\title{
Development of $\gamma$-Oryzanol Rich Extract from Leum Pua Glutinous Rice Bran Loaded Nanostructured Lipid Carriers for Topical Delivery
}

\author{
Warangkana Pornputtapitak ${ }^{1 *}$, Jaturavit Pantakitcharoenkul ${ }^{1}$, Ratchada Panpakdee ${ }^{1}$, \\ Veerawat Teeranachaideekul ${ }^{2}$ and Nuttanan Sinchaipanid ${ }^{3}$ \\ ${ }^{1}$ Department of Chemical Engineering, Faculty of Engineering, Mahidol University, 25/25 Phutthamonthon 4 Road, Salaya, Nakhon Pathom \\ 73170, THAILAND \\ ${ }^{2}$ Department of Pharmacy, Faculty of Pharmacy, Mahidol University, 447 Sri-Ayuthaya Rajathevi Bangkok 10400, THAILAND \\ ${ }^{3}$ Department of Manufacturing Pharmacy, Faculty of Pharmacy, Mahidol University, 447 Sri-Ayuthaya Rajathevi Bangkok 10400, THAILAND
}

\begin{abstract}
Leum Pua is native Thai glutinous rice that contains antioxidants higher than white rice and other colored rice. One of the major antioxidants in rice brans is $\gamma$-oryzanol (GO). In this study, Leum Pua glutinous rice bran was extracted by different solvents. Oleic acid $(\sim 40 \mathrm{~g} / 100 \mathrm{~g}$ extract $)$, linoleic acid $(\sim 30$ $\mathrm{g} / 100 \mathrm{~g}$ extract), and palmitic acid ( $\sim 20 \mathrm{~g} / 100 \mathrm{~g}$ extract) were found to be major lipid components in the extracts. Methanol extract showed less variety of lipid components compared to the others. However, hexane extract showed the highest percent of $\gamma$-oryzanol compared to other solvents. Therefore, the hexane extract was selected to prepare nanostructured lipid carriers (NLC). The prepared NLC had small particles in the size range of $142.9 \pm 0.4 \mathbf{n m}$ for extract-loaded NLC and $137.1 \pm 0.5 \mathbf{n m}$ for GO-loaded NLC with narrow size distribution $(\mathrm{PI}<0.1)$ in both formulations. The release profile of extract-loaded NLC formulation was slightly higher than GO-loaded NLC formulation. However, they did not follow the Higuchi model because of small amounts of $\gamma$-oryzanol loaded in NLC particles.
\end{abstract}

Key words: $\gamma$-oryzanol, solvent extraction, nanostructured lipid carriers, glutinous rice bran, Leum Pua

\section{INTRODUCTION}

Lipid nanoparticles are developed for two decades as an alternative carrier to traditional carriers such as liposomes and polymeric nanoparticles ${ }^{1}$. Lipid nanoparticles are prepared in a form of solid lipid nanoparticles (SLN) for the first generation and then nanostructured lipid carriers (NLC) is invented for the second generation to overcome the limitations of SLN. The difference between SLN and NLC is the liquid lipid content that is added in NLC, whereas only solid lipid is used to prepare SLN. Consequently, amorphous clusters are presented in NLC compared to crystal lattice in SLN. The crystallinity of lipid particles directly affects the drug loading capacity and the drug expulsion during storage. The literature reported increase in drug loading of NLC compared to $\mathrm{SLN}^{2}$. Furthermore, the crystallinity and the structure in particle matrix of NLC can be controlled by the mixing of liquid lipid and solid lipid contents. Another advantage of NLC includes drug release profiles of active ingredients loaded in lipid nanoparticles. SLN provide burst release at the initial time due to the phase separation and a large amount of drug at the outer shell. In addition, the fast transformation to high order polymorphs also causes drug expulsion in SLN. Meanwhile, NLC can be generated imperfect forms and amorphous forms so that it can provide higher flexibility for the desired prolonged release profiles ${ }^{3)}$.

$\gamma$-Oryzanol (GO) is a natural component found in rice bran and rice germ. It consists of a mixture of ferulic acid esters of plant sterols and triterpene alcohols ${ }^{4}$. The five major components are cycloartenyl ferulate, 24-methylene cycloartanyl ferulate, campesteryl ferulate, $\beta$-sitosteryl ferulate and cycloartanyl ferulate ${ }^{4)}$. However, at least 10 components were reported for $\gamma$-oryzanol ${ }^{5)}$. The $\gamma$-oryzanol is an antioxidant compound, which is excellent in inhibiting lipid peroxidation ${ }^{6)}$. Furthermore, it exhibits some healthbeneficial properties such as increasing high-density lipoproteins level while decreasing total plasma cholesterol ${ }^{7}$. The $\gamma$-oryzanol is also used in food applications for improv-

\footnotetext{
*Correspondence to: Warangkana Pornputtapitak, Department of Chemical Engineering, Faculty of Engineering, Mahidol University, 25/25 Phutthamonthon 4 Road, Salaya, Nakhon Pathom 73170, THAILAND

E-mail: Warangkana.por@mahidol.ac.th, beauvp9@gmail.com

Accepted September 4, 2017 (received for review May 9, 2017)

Journal of Oleo Science ISSN 1345-8957 print / ISSN 1347-3352 online

http://www.jstage.jst.go.jp/browse/jos/ http://mc.manusriptcentral.com/jjocs
} 
ing food stability ${ }^{8}$ and pharmaceutical applications for UV-A filter in sunscreen cosmetics ${ }^{9)}$. Since $\gamma$-oryzanol is a poor water soluble compound, the utility of this compound is limited. Therefore, applying $\gamma$-oryzanol into lipid nanoparticles is an alternative for any applications, especially for topical delivery.

Leum Pua is native Thai glutinous rice that got seed certification for genetic purity in 2012. Leum Pua rice is a purple rice with high nutrition value. Normally, colored rice has two to three times higher of the amount of anthocyanin, $\gamma$-oryzanol, and phenolic compounds than white rice ${ }^{10)}$. Rice Research and Development Division(Rice Department, Ministry of Agriculture and Cooperatives of Thailand)also reported that Leum Pua rice has higher antioxidant than other colored rice. Its grain contains anthocyanin, $\gamma$-oryzanol, omega-3, omega-6, omega-9, $\alpha$-tocopherol, zinc, calcium, manganese and other antioxidants. The development of $\gamma$-oryzanol rich extract loaded NLC for topical delivery can lead to value added of native Thai glutinous rice. In this study, Leum Pua rice bran was extracted by using different solvents. $\gamma$-Oryzanol, $\alpha$-tocopherol and lipid compositions in each extract were analyzed. The best extract was, then, selected for loading in NLC particles. Prepared NLC formulations were characterized by photon correlation spectroscopy (PCS) and differential scanning calorimetry (DSC). The encapsulation efficiency and the release profile were also investigated and compared to GO-loaded NLC formulation.

\section{EXPERIMENTAL}

\subsection{Materials}

Cetyl palmitate and caprylic/capric triglycerides were purchased from Namsiang Group (Bangkok, Thailand). $\gamma$-Oryzanol, Tween80 (polysorbate 80) and Span40 (sorbitan monopalmitate) were purchased from Sigma-Aldrich (Japan). Hexane, isopropanol, and methanol were purchased from RCI Labscan (Bangkok, Thailand). Leum Pua glutinous rice bran was provided by a local rice mill in the northeast of Thailand. Distilled water and deionized water were prepared freshly from Autostil ${ }^{\mathrm{TM}}$ 8000x (England) and aquaMax $^{\text {TM }}$-Ultra (Korea), respectively. Phosphate buffer saline(PBS) pH 5.5 was prepared freshly in Laboratory. All other chemicals were commercially available in analytical grade. The materials were used without further purification procedures.

\subsection{Extraction of oil from Leum Pua glutinous rice bran}

Twenty grams of Leum Pua glutinous rice bran was extracted with $300 \mathrm{ml}$ of different solvents (hexane, isopropanol, and methanol) by using Soxhlet extraction. Then, the solvents were evaporated under reduced pressure by using Rotavapor (Buchi Rotavapor ${ }^{\circledR}$ R-210/R251) to obtain rice bran oil extract.

\subsection{Preparation of NLC formulation}

Nanostructured lipid carriers (NLC) formulations were prepared by hot high-pressure homogenization method ${ }^{22)}$. Lipid phase consisted of solid lipid (cetyl palmitate), liquid lipid (caprylic/capric triglycerides) and surfactant (span40) were melted together at $80^{\circ} \mathrm{C}$ and then mixed with aqueous phase contained Tween80 in water under homogenizer (ULTRA-TURRAX $^{\circledR}$ T25, IKA) at $8000 \mathrm{rpm}$ for $1 \mathrm{~min}$ to become pre-emulsions. Then, pre-emulsions were continuously heated to maintain the temperature at $80^{\circ} \mathrm{C}$ before they were processed by a high-pressure homogenizer (High-Pressure Homogenizer ${ }^{\circledR}$ APV-2000). The pre-emulsions were applied for 3 cycles at 500 bars and around $80^{\circ} \mathrm{C}$. The prepared NLC formulations were cooled down at ambient conditions to room temperature. $0.2 \% \mathrm{w} / \mathrm{w}$ of DMDM hydantoin was added to cooled formulations as a preservative.

In the case of active ingredient loaded NLC, $1 \% \mathrm{w} / \mathrm{w}$ of hexane extract, $0.01 \% \mathrm{w} / \mathrm{w}$ and $1 \% \mathrm{w} / \mathrm{w}$ of standard $\gamma$-oryzanol were added separately to the lipid phase before pre-emulsion preparation step. The amount of active ingredient loaded NLC calculated based on total weight of the formulation. The compositions of each formulation were shown in Table 1.

\subsection{Particle size measurement}

Particle size analysis was performed by photon correlation spectroscopy (PCS) using Malvern Zetasizer Nano-ZS (Malvern Instruments, UK). $173^{\circ}$ scattering angle was applied at room temperature. Samples were diluted 10 times with deionized water before measuring in the folded capillary cell(DTS1060). The mean particle size and the

Table 1 The compositions of NLC $(\% \mathrm{w} / \mathrm{w})$ formulation.

\begin{tabular}{lcccccc}
\hline Formulation & $\begin{array}{c}\text { Cetyl } \\
\text { palmitate }\end{array}$ & $\begin{array}{c}\text { Caprylic/capric } \\
\text { triglycerides }\end{array}$ & Tween80 & Span40 & Active ingredient & Water q.s. \\
\hline GO-loaded NLC & 7 & 3 & 2 & 2 & $\begin{array}{l}0.01 \% \text { w/w of standard } \\
\gamma \text {-oryzanol }\end{array}$ & 100 \\
Extract-loaded NLC & 7 & 3 & 2 & 2 & $\begin{array}{l}1 \% \mathrm{w} / \mathrm{w} \text { of hexane } \\
\text { extract }\end{array}$ & 100 \\
\hline
\end{tabular}


polydispersity index (PI) of each formulation were obtained by the average of 5 measurements. All measurements were done in triplicate.

\subsection{Differential scanning calorimetry (DSC)}

The degree of crystallinity of NLC formulations was performed by using a differential scanning calorimeter (DSC Q200 TA Instruments). Sufficient amount of NLC formulations was weighed and put in a $40 \mu \mathrm{l}$ aluminum pan. An empty aluminum pan was used as the reference. The samples were scanned from $00^{\circ} \mathrm{C}$ to $85^{\circ} \mathrm{C}$ with the heating rate of $5{ }^{\circ} \mathrm{C} / \mathrm{min}$ and from $85^{\circ} \mathrm{C}$ to $0^{\circ} \mathrm{C}$ with the same cooling rate for 2 cycles. The melting point temperature, the melting onset temperature, and the melting enthalpy were evaluated. The crystallinity index (\% CI) was calculated by using the following equation.

$$
\% \mathrm{CI}=\frac{\Delta H_{\text {NLC aqueous dispersion }(J g)}}{\Delta H_{\text {bulk material }(J g)} \times \text { Concentration }_{\text {lipid phase }(\%)}} \times 100
$$

\subsection{Drug encapsulation efficiency (\%EE)}

The encapsulation efficiency (\%EE) of $\gamma$-oryzanol in NLC formulation was indirectly evaluated by centrifugal filtration. Four grams of NLC formulations were placed into a centrifugal filter tube (Amicon ${ }^{\circledR}$ Ultra-15, $30 \mathrm{kDa}$ molecular weight cut-off, Millipore, Ireland) and centrifuged at $5000 \mathrm{~g}$ for $30 \mathrm{~min}$ at room temperature. The supernatant was examined the amount of $\gamma$-oryzanol by using reverse-phase high-pressure liquid chromatography (RP-HPLC) .

\subsection{High performance liquid chromatography (HPLC)}

HPLC was performed by using Waters Alliance 2695 HPLC Separations, and Waters ${ }^{\circledR} 2489$ UV/Visible detector. The Sunfire C18 column with $4.6 \times 100 \mathrm{~mm}$ diameter $(3.5$ $\mu \mathrm{m})$ was applied. The mobile phase was a mixture of acetonitrile, methanol, and isopropanol $(45: 30: 25 \%(\mathrm{v} / \mathrm{v} / \mathrm{v}))$. The sample was detected at the wavelength of $325 \mathrm{~nm}$ and run time of $20 \mathrm{~min}$, with a flow rate of $0.8 \mathrm{ml} / \mathrm{min}$ and $20 \mu \mathrm{ln}-$ jection volume. The samples from the extraction were prepared in acetone, whereas the samples from release study were in a release study medium (a mixture of phosphate buffer saline (PBS), pH 5.5, 50\% (v/v) isopropanol and 5\% $(\mathrm{w} / \mathrm{w})$ Tween 80$)$.

\subsection{In vitro release study}

In vitro release study was performed by using static Franz diffusion cells to determine the amount of $\gamma$-oryzanol released from prepared formulations. Mixed cellulose ester membrane filters with a pore size diameter of $0.1 \mu \mathrm{m}$ (Advantec ${ }^{\circledR}$, Japan) were used as release membrane. The surface area of the release membrane was $1.60-2.40 \mathrm{~cm}^{2}$ depended on the Franz diffusion cell. The experiments were operated at the controlled temperature of $32^{\circ} \mathrm{C}$ to mimic human skin. The receptor medium was $10 \mathrm{ml}$ of a mixture of phosphate buffer saline(PBS), pH 5.5 and 50\% $(\mathrm{v} / \mathrm{v})$ isopropanol and $5 \%(\mathrm{w} / \mathrm{w})$ Tween 80 . The medium was stirred by a magnetic bar during the experiment to make a receptor medium homogeneous and minimize stagnant layers. $300 \mu \mathrm{l}$ of NLC formulation was loaded onto the membrane in the donor compartment. $0.5 \mathrm{ml}$ of a receptor medium was collected at suitable time intervals over $24 \mathrm{~h}$ using a syringe needle, and the same volume of the medium was replaced with freshly receptor medium. The collected samples were analyzed by HPLC method as previously described. The experiment was performed in triplicate. The data were presented as the mean values \pm standard deviation (SD).

\subsection{Statistical analysis}

The data were expressed as the mean values \pm standard deviation (SD) for particle size measurements. Statistical significance of difference was examined using one-way ANOVA at the probability level of 0.05 .

\section{RESULT AND DISCUSSION}

\subsection{Yields and compositions of Leum Pua glutinous rice bran extract}

Native Thai glutinous rice bran called "Leum Pua" was extracted by using different solvents. The percent yield of the extract from each solvent was shown in Table 2. The results presented that methanol gave the highest percent of the extract $(33.05 \% \mathrm{w} / \mathrm{w}$ of rice bran) compared to isopropanol $(19.35 \% \mathrm{w} / \mathrm{w}$ of rice bran) and hexane $(13.50 \% \mathrm{w} /$ $\mathrm{w}$ of rice bran), respectively. However, the precipitation was observed when the extract was kept for a while. The methanol extract showed the most precipitation compared

Table 2 The percent yields and the phytochemical compositions of the extracts when using methanol, isopropanol, and hexane as extraction solvents.

\begin{tabular}{cccc}
\hline Extraction solvent & $\begin{array}{c}\text { Extraction yield } \\
(\mathrm{g} / 100 \mathrm{~g} \text { rice bran })\end{array}$ & $\begin{array}{c}\gamma \text {-Oryzanol } \\
(\mathrm{g} / 100 \mathrm{~g} \text { extract })\end{array}$ & $\begin{array}{c}\alpha \text {-Tocopherol } \\
(\mathrm{mg} / 100 \mathrm{~g} \text { extract })\end{array}$ \\
\hline Methanol & 33.05 & 0.74 & 8.99 \\
Isopropanol & 19.35 & 0.52 & 10.04 \\
Hexane & 13.50 & 0.97 & 7.84 \\
\hline
\end{tabular}


to the others. This could be explained by high polarity of methanol over other solvents so that methanol dissolved many compounds, especially high polar compounds. When the solvent was removed, less soluble compounds were precipitated out of the extract.

The lipid compositions of the extracts were analyzed by in house method based on the compendium of methods for food analysis, Thailand (2003) (Table 3). The fatty acids, which predominated in rice bran oil extracted by each solvent, were oleic acid (around $40 \mathrm{~g} / 100 \mathrm{~g}$ extract), linoleic acid (around $30 \mathrm{~g} / 100 \mathrm{~g}$ extract), and palmitic acid (around $20 \mathrm{~g} / 100 \mathrm{~g}$ extract), respectively. Different species of rice bran had different percents of fatty acids in the extracts. However, these three main fatty acids were found to be the major components in rice bran oils ${ }^{11,12)}$. It was noteworthy that methanol extract had fewer lipid compositions than isopropanol extract and hexane extract because of the high polarity of methanol.

$\gamma$-Oryzanol was examined by using RP-HPLC and $\alpha$-tocopherol was analyzed by in house method based on BS EN 12823-1:2000. The amount of $\gamma$-oryzanol and $\alpha$-tocopherol were shown in Table 2. The results indicated $\alpha$-tocopherol was higher in isopropanol extract than methanol extract and hexane extract, respectively. In contrast to $\alpha$-tocopherol, $\gamma$-oryzanol was highest in hexane extract compared to methanol extract and isopropanol extract. This study was consistent with Chen's studies that reported the potential of methanol over hexane for extracting the Taro-Scented Japonica rice bran ${ }^{13)}$. They also mentioned that methanol had high extraction efficieney in total phenolic substances, oryzanol, and tocols. Although the higher tocols content was reported for methanol extract, they found that the oryzanol content was higher in hexane extract compared to methanol extract. Other studies mentioned isopropanol as an alternative solvent for extracting oil from rice $\operatorname{bran}^{14,15)}$. Similar to this study, isopropanol was better to extract vitamin $\mathrm{E}$, but hexane was better to extract oryzanol.

Although these studies used different extraction condition and extraction method, as well as different rice species, the effect of solvents was similar. This indicated that the properties of solvent such as the polarity and the structure of solute, directly affected the phytochemical compositions in rice bran extract ${ }^{11}$. $\alpha$-Tocopherol molecule contains an alkyl side chain and a hydroxyl group which attached to a benzene ring and an oxygen atom attached at para-position to another oxygen atom. On the contrary, the molecular structures of $\gamma$-oryzanol are sterols. Therefore, from the molecular structure, $\alpha$-tocopherol is less hydrophobic than $\gamma$-oryzanol ${ }^{16)}$. Methanol and isopropanol are polar protic solvents whereas hexane is nonpolar solvent. Thas, $\alpha$-tocopherol is compatible with methanol and isopropanol more than hexane whereas $\gamma$-oryzanol is preferable to hexane.

\subsection{Characterization of solvent extracts}

$\gamma$-Oryzanol in the extract was examined by using RP-HPLC as previously described. The HPLC chromatogram of $\gamma$-oryzanol displayed three major peaks and two minor peaks at retention time of around 11, 12, 13, 14 and 16 min, respectively. The HPLC chromatogram of $\gamma$-oryzanol in hexane extract was shown in Fig. 1. In comparison with literature, three main peaks at $11.124 \mathrm{~min}$ (21.18\%), $11.894 \min (51.39 \%)$ and $12.949 \min (15.77 \%)$

Table 3 The lipid compositions of the extracts.

\begin{tabular}{llccc}
\hline \multicolumn{1}{c}{$\begin{array}{c}\text { Fatty acid } \\
\text { (g/100 g sample })\end{array}$} & & Hexane extract & Isopropanol extract & Methanol extract \\
\hline Saturated fatty acid & & & & \\
Myristic acid & $\mathrm{C} 14: 0$ & 0.57 & 0.57 & - \\
Palmitic acid & $\mathrm{C} 16: 0$ & 19.42 & 19.61 & 20.51 \\
Stearic acid & $\mathrm{C} 18: 0$ & 2.11 & 2.23 & 2.25 \\
Arachidic acid & $\mathrm{C} 20: 0$ & 0.69 & 0.67 & 0.54 \\
Behenic acid & $\mathrm{C} 22: 0$ & 0.23 & 0.21 & - \\
Lignoceric acid & $\mathrm{C} 24: 0$ & 0.38 & 0.38 & - \\
& & & & - \\
Unsaturated fatty acid & & & & - \\
Palmitoleic acid & $\mathrm{C} 16: 1$ & 0.19 & 0.20 & - \\
Oleic acid & $\mathrm{C} 18: 1 \mathrm{n} 9 \mathrm{c}$ & 41.10 & 40.73 & -1.28 \\
Cis-11-Eicosenoic acid & $\mathrm{C} 20: 1 \mathrm{n} 11$ & 0.41 & 0.40 & 1.02 \\
Linoleic acid & $\mathrm{C} 18: 2 \mathrm{n} 6 \mathrm{c}$ & 29.37 & 29.47 & \\
$\alpha$-linolenic acid & $\mathrm{C} 18: 3 \mathrm{n} 3$ & 1.15 & 1.13 & \\
\hline
\end{tabular}




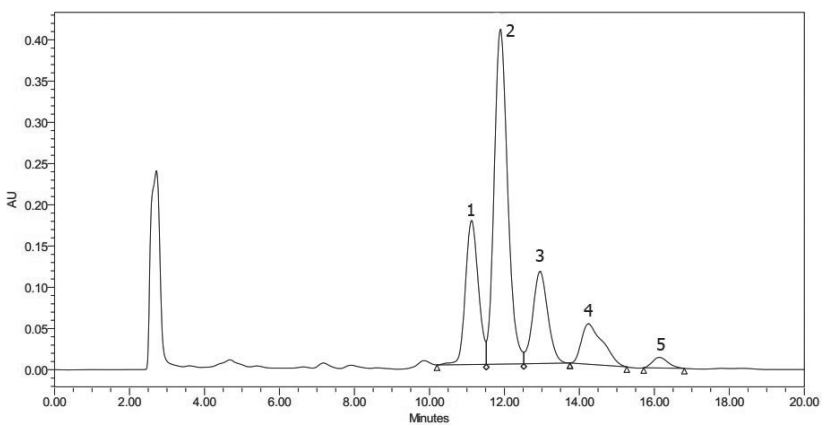

Fig. 1 The HPLC chromatogram of $\gamma$-oryzanol in hexane extract.

were identified to be cycloartenyl ferulate, 24-methylene cycloartanyl ferulate, and campesteryl ferulate, respectively. These three main peaks were taken around $85 \%$ of $\gamma$-oryzanol. This was consistent with the report of $80 \%$ of $\gamma$-oryzanol in normal rice bran ${ }^{17)}$. The peak at $14.241 \mathrm{~min}$ with the area under the curve of $9.8 \%$ was identified as the combination of $\beta$-sitosteryl ferulate and cycloartanyl ferulate ${ }^{4)}$. The smallest peak at 16.135 min with only $1.87 \%$ of peak area might be identified as sitostanyl ferulate ${ }^{5)}$. The amount of $\gamma$-oryzanol was calculated by summation of the area under the curve of these five peaks. The peak at around 2.75 min was a peak of acetone that was used as a solvent for sample preparations.

\subsection{Characterization of NLC formulations}

NLC formulations were prepared by using high-pressure homogenization ( $\mathrm{HPH}$ ) method. They were composed of $10 \%(\mathrm{w} / \mathrm{w})$ lipid contents and $4 \%(\mathrm{w} / \mathrm{w})$ of emalsifiers. Different types of lipid were preliminary studied. However, cetyl palmitate was used as solid lipid and caprylic/capric triglyceride was used as liquid lipid. Tween80 (hydrophilic surfactant) and Span40 (hydrophobic surfactant) were used as emalsifiers in the ratio of 1 to 1 . Then, $1 \%(\mathrm{w} / \mathrm{w})$ of hexane extract was loaded to the NLC by adding the extract to lipid phase before preparing pre-emulsion as described previously. Since the results in Table 2 showed the highest percent of $\gamma$-oryzanol in hexane extract, the hexane extract was chosen to prepare NLC formulation. The formulation was compared to $0.01 \%$ (w/w) GO-loaded NLC. The amount of $\gamma$-oryzanol that was added to the formulation was calculated based on the amount of $\gamma$-oryzanol in the hexane extract from the previous section(Table 2). The mean particle size, the polydispersity index (PI) and the zeta potential of extract-loaded NLC and GO-loaded NLC were shown in Table 4.

Extract-loaded NLC had a larger mean particle size (142.9 $\pm 0.4 \mathrm{~nm})$ compared to GO-loaded NLC $(137.1 \pm 0.5$ $\mathrm{nm})$ at $95 \%$ of confidence $(p<0.05)$. The high quality of particle dispersion was performed by the PI of both formulations that was smaller than 0.1 . The PI of extract-loaded NLC was $0.074 \pm 0.006$ and the PI of GO-loaded NLC was $0.090 \pm 0.019$. The PI demonstrates particle size distribution. The lower the PI, the better the quality of dispersion and the closer the monodispersity. The PI normally ranges from 0 to 1 . The acceptable PI should be lower than 0.5 to indicate the narrow size distribution and it should be lower than 0.1 for the excellent quality of dispersion ${ }^{18)}$. Other studies also suggested the lower or equal to 0.3 for optimum $\mathrm{PI}^{19)}$. In this study, GO-loaded NLC formulation and extract-loaded NLC formulation showed the monodispersity with the PI lower than 0.1 .

Besides PI, zeta potential of both formulations were not significantly different $(p>0.05)$. Although the zeta potential was not a primary parameter for considering the quality of the formulation, it implied to the physical stability of particles. Zeta potential involves with surface charges of particles that can be positive or negative. High zeta indicates high repulsive force, resulting in prevention of particle aggregation. In general, nanoparticles with good physical stability should have zeta potential higher than +20 $\mathrm{mV}$ or lower than $-20 \mathrm{mV}$. However, some studies mentioned that this was not always true and the stability studies should be established ${ }^{20)}$. The zeta potentials of GOloaded NLC and extra-loaded NLC formulation were - 26.9 $\pm 1.0 \mathrm{mV}$ and $-26.1 \pm 0.9 \mathrm{mV}$, respectively (Table 4 ). Thus, these formulations should be stable on storage. The formulations were kept at $4^{\circ} \mathrm{C}$ and room temperature for 12 weeks to check for the storage physical stability.

The data in Table 5 indicated the physical stability of NLC formulations when they were stored at $4{ }^{\circ} \mathrm{C}$ and room temperature for 12 weeks. Further study found that nanoparticles that had a small mean particle size such as in the range of $100-200 \mathrm{~nm}$, and had a low PI were physically stable for long time storage. This could be explained by the physical properties of particles in the nanosized range that normally disperses in nature. Moreover, it was noteworthy that the particle size of the NLC formulations showed slightly lower when kept at room temperature compared to $4^{\circ} \mathrm{C}(p<0.05)$. The mean particle size also

Table 4 The mean size, the polydispersity index and the zeta potential of extract-loaded NLC formulation and GO-loaded NLC formulation.

\begin{tabular}{lccc}
\hline \multicolumn{1}{c}{ Formulation } & $\begin{array}{c}\text { Mean particle size } \\
(\mathrm{nm})\end{array}$ & $\begin{array}{c}\text { Polydispersity index } \\
(\mathrm{PI})\end{array}$ & Zeta potential \\
\hline GO-loaded NLC & $137.1 \pm 0.5$ & $0.090 \pm 0.019$ & $-26.9 \pm 1.0$ \\
Extract-loaded NLC & $142.9 \pm 0.4$ & $0.074 \pm 0.006$ & $-26.1 \pm 0.9$ \\
\hline
\end{tabular}


W. Pornputtapitak, J. Pantakitcharoenkul, R. Panpakdee et al.

Table 5 The mean particle size of extract-loaded NLC formulation and GO-loaded NLC formulation stored at $4^{\circ} \mathrm{C}$ and room temperature for 12 weeks.

\begin{tabular}{cccccc}
\hline \multirow{2}{*}{ Formulation } & \multicolumn{5}{c}{ Particle size $(\mathrm{nm})$} \\
\cline { 2 - 6 } & Week 0 & Week 6 & Week 12 & Week 6 & Week 12 \\
\cline { 2 - 6 } & $137.1 \pm 0.5$ & $136.4 \pm 0.5$ & $131.9 \pm 1.6$ & $127.9 \pm 0.7$ & $123.8 \pm 2.3$ \\
\hline GO-loaded NLC & $142.9 \pm 0.4$ & $141.5 \pm 2.2$ & $138.4 \pm 1.3$ & $136.4 \pm 1.4$ & $135.3 \pm 1.3$ \\
\hline Extract-loaded NLC & 142.4 &
\end{tabular}

Table 6 DSC parameters of NLC formulations.

\begin{tabular}{lcccc}
\hline \multicolumn{1}{c}{ Formulation } & $\begin{array}{c}\text { Melting onset } \\
\left({ }^{\circ} \mathrm{C}\right)\end{array}$ & $\begin{array}{c}\text { Melting point } \\
\left({ }^{\circ} \mathrm{C}\right)\end{array}$ & $\begin{array}{c}\text { Enthalpy change } \\
(\mathrm{J} / \mathrm{g})\end{array}$ & $\% \mathrm{CI}$ \\
\hline Cetyl palmitate & 48.59 & 50.93 & 226.7 & 100 \\
Drug-free NLC & 42.21 & 44.01 & 7.858 & 50 \\
GO-loaded NLC & 41.30 & 43.81 & 7.113 & 45 \\
Extract-loaded NLC & 40.95 & 44.79 & 9.157 & 58 \\
\hline
\end{tabular}

slightly decreased when NLC formulations were kept for 6 weeks and 12 weeks, especially for GO-loaded NLC formulation $(p<0.05)$.

A study also mentioned that low zeta potential could be related to high storage stability due to the perfect coverage of non-ionic surfactants. This was possible due to the stability of these lipid nanoparticles influenced by a steric stabilization instead of an electrostatic stabilization. They also referred that coverage of non-ionic surfactants on the lipid nanoparticle surface decreased the mobility of nanoparticles, resulting in lower zeta potential value ${ }^{21}$. Tween 80 and Span40 are non-ionic surfactants that were selected for prepared NLC formulations in this study. Thus, not only did the zeta potential suggest the physical stability of NLC, but the non-ionic surfactant in the system also supported the stability of the NLC.

\subsection{DSC investigations}

The degree of crystallinity of the NLC was performed by differential scanning calorimetry (DSC). The melting onset, the melting point, the enthalpy change and the percent of crystallinity (\% CI) were determined (Table 6). The data showed that the melting point and the melting onset of NLC formulations were lower than pure cetyl palmitate. The melting enthalpy decreased compared to the pure cetyl palmitate, resulting in lower of the degree of crystallinity. The DSC thermogram of cetyl palmitate revealed a main endothermic peak at $50.93^{\circ} \mathrm{C}$ and a smaller peak at around $44^{\circ} \mathrm{C}$ indicated $\beta$ polymorphic form which was the most stable form of cetyl palmitate and $\alpha$ polymorphic form of cetyl palmitate, respectively. In general, triglycerides can crystallize in three polymorphic forms, which are $\alpha$-form, $\beta^{\prime}$-form and $\beta$-form. The $\beta$-form is the stable form that is a main melting peak whereas the $\alpha$-form shows as a small endothermic peak with low melting enthalpy ${ }^{22}$. The $\alpha$-form has the highest Gibbs free energy because of disordered aliphatic chains. The melting enthalpy of $\alpha$-form appears at a lower temperature than the main peak and tends to transit to the stable $\beta$-form during storage. Although the $\beta$-form is the most stable due to the lowest Gibbs free energy, some mixed-acid triglycerides were reported that the $\beta$ '-form was the most stable form ${ }^{23)}$. In this study, NLC formulations were prepared by mixing cetyl palmitate with liquid lipid. The DSC thermograms of NLC formulations showed melting peaks shifted to lower temperature compared to cetyl palmitate. Not only had the lower melting enthalpy of the main melting peak, but it also had a little shoulder in the main melting peak (Fig. 2). This could be referred to a metastable form ( $\beta^{\prime}$-form) of cetyl palmitate in NLC formulations ${ }^{24)}$. The metastable $\beta^{\prime}$ form was also mentioned to have a higher capacity for drug loading compared to the stable form ${ }^{25)}$. In addition, $\gamma$-oryzanol had endothermic melting peaks around $60-80^{\circ} \mathrm{C}$ (data not shown). However, it could not be seen on the DSC thermograms of NLC formulations. This might be due to the dissolution of $\gamma$-oryzanol in the lipid composition, especially the liquid lipid components.

When considering the DSC thermogram of NLC formulations (Fig. 2), it showed that drug-free NLC and GO-loaded NLC were almost similar, but extract-loaded NLC formulation showed a wider endothermic peak with several shoulders and side maxima. The data on Table 6 indicated the wider range of melting temperature of extract-loaded NLC formulation compared to the other formulations. Moreover, it also had a small endothermic peak around $32^{\circ} \mathrm{C}$. DSC was run for two cycles. Several crystallization peaks were found on the DSC thermogram of extract-loaded NLC formulation when the sample was cooled back down to $0^{\circ} \mathrm{C}$ due to the mixed lipid in the extract as mentioned previously (Table 2). These results implied that the different 


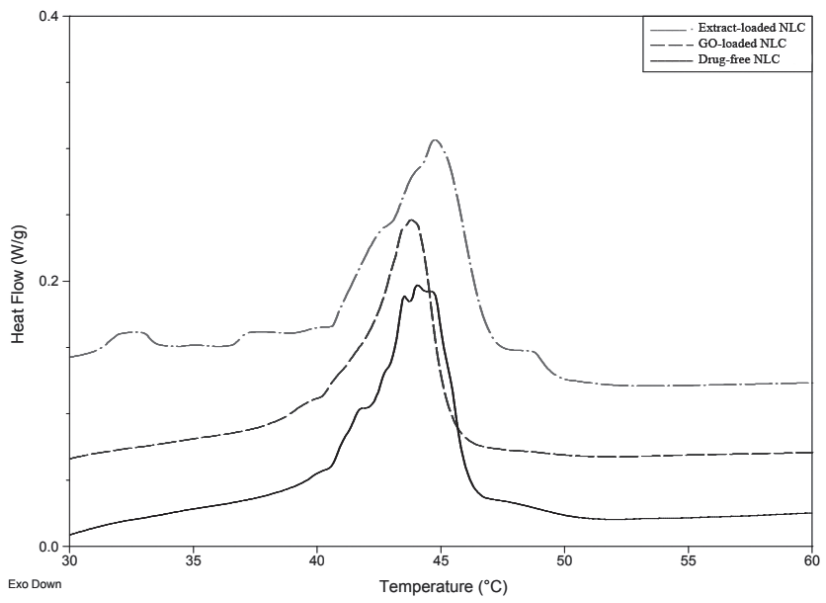

Fig. 2 The DSC thermogram of extract-loaded NLC formulation compared to GO-loaded NLC formulation and drug-free NLC formulation.

crystallization of mixed lipids in the extract might affect the drug loading and the release of the drug molecule from the NLC formulation.

\subsection{Encapsulation efficiency}

NLC formulations were analyzed the percent of encapsulation efficiency (\%EE) by using RP-HPLC. $\gamma$-Oryzanol is lipophilic drug that solubilizes better in lipid phase than in aqueous phase. Therefore, most $\gamma$-oryzanol should be encapsulated in NLC particles. In this study, $1 \% \mathrm{w} / \mathrm{w}$ of hexane extract was loaded in the NLC formulation. According to the percent of $\gamma$-oryzanol in hexane extract was around $1 \% \mathrm{w} / \mathrm{w}$, only $0.01 \% \mathrm{w} / \mathrm{w}$ of standard $\gamma$-oryzanol was loaded to NLC formulation for the comparison. Nevertheless, $1 \% \mathrm{w} / \mathrm{w}$ GO-loaded NLC formulation was also prepared to confirm the efficiency of NLC formulation for $\gamma$-oryzanol encapsulation. The data exhibited that most $\gamma$-oryzanol was loaded in the NLC formulation, although $1 \% \mathrm{w} / \mathrm{w}$ of $\gamma$-oryzanol was applied in the formulation. The $\%$ EE of extract-loaded NLC, $0.01 \% \mathrm{w} / \mathrm{w}$ and $1 \% \mathrm{w} / \mathrm{w}$ GO-loaded NLC were $99.944 \pm 0.019 \%, 100 \%$ and 99.936 $\pm 0.019 \%$, respectively. For $0.01 \%$ w/w GO-loaded NLC, the amount of $\gamma$-oryzanol in the ultrafiltrate could not be detected by HPLC, so the \% EE was assumed to be $100 \%$.

\subsection{In vitro release studies}

In vitro release studies were performed by using static vertical Franz diffusion cells to determine the amount of $\gamma$-oryzanol released through the artificial membrane per time. The acceptor medium was controlled at $32^{\circ} \mathrm{C}$ and $\mathrm{pH}$ 5.5 in order to mimic the human skin surface condition. The medium was collected at 2, 4, 6, 8, 10, 12, and $24 \mathrm{~h}$ and analyzed by using RP-HPLC at $325 \mathrm{~nm}$. $\gamma$-Oryzanol composed of five peaks (Fig. 1). The combination of those peaks was counted for the amount of $\gamma$-oryzanol. Sink con-

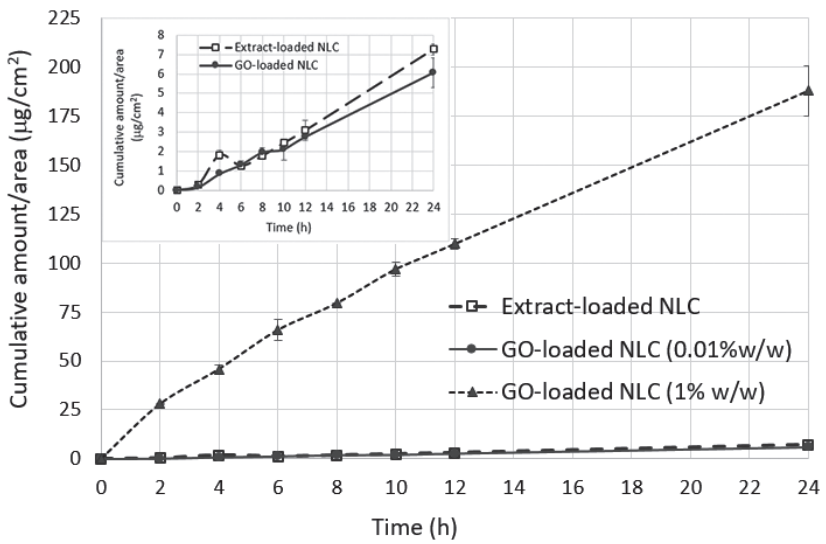

Fig. 3 Cumulative amount per unit area $\left(\mu \mathrm{g} / \mathrm{cm}^{2}\right)$ of $\gamma$-oryzanol released from NLC over $24 \mathrm{~h}$ of extractloaded NLC formulation and $0.01 \% \mathrm{w} / \mathrm{w}$ GO-loaded NLC formulation compared to $1 \% \mathrm{w} / \mathrm{w}$ GO-loaded NLC formulation. The small figure on the top left was shown to expand the cumulative amount per unit area $\left(\mu \mathrm{g} / \mathrm{cm}^{2}\right)$ of $\gamma$-oryzanol released from NLC over $24 \mathrm{~h}$ of extract-loaded NLC formulation compared to $0.01 \% \mathrm{w} / \mathrm{w}$ GO-loaded NLC formulation.

ditions were maintained over the experiments. The release profile of extract-loaded NLC formulation was compared to that of GO-loaded NLC formulation(Fig. 3). The $\gamma$-oryzanol in extract-loaded NLC formulation tended to release slightly higher than GO-loaded NLC formulation. This could be explained by the higher amount of oil phase in extractloaded NLC formulation. Moreover, as mentioned previously, the different recrystallization peaks were presented on the DSC thermogram of extract-loaded NLC formulation. The crystallization of mixed lipids could be interrupted the crystallization of polymorphic forms of cetyl palmitate which was the main solid lipid in the NLC formulation. Consequently, this formulation should be in amorphous state more than the formulation that had only two lipid compartments. Several studies also proposed that NLC provided drug loading capacity higher than SLN because NLC had amorphous clusters whereas SLN had crystal lattices in the particles. The relatively perfect crystal lattice in SLN limited the space for drugs accommodated in the particles $^{2)}$. Therefore, the amorphous state was preferable for nanocarriers such as NLC. However, if the release profiles of both extract-loaded NLC formulation and GO-loaded NLC formulation $(0.01 \% \mathrm{w} / \mathrm{w} \gamma$-oryzanol) were compared to the GO-loaded NLC formulation ( $1 \% \mathrm{w} / \mathrm{w} \gamma$-oryzanol), these two release profiles were almost similar due to the large amount of $\gamma$-oryzanol in 1\% w/w GO-loaded NLC formulation(Fig. 3).

In general, the release profile of drugs from NLC and SLN contained two states. The initial state was the faster release owing to the accumulation of liquid lipid at the 
outer shell of lipid nanoparticles after crystallization of solid lipid. The liquid lipid at the outer shell directly related to the amount of lipophilic drug that dissolved in liquid lipid at the outer shell. Hence, the release profile normally showed faster release at the initial state. The second state was the prolonged release, which caused by slower diffusion of the drug from the inside of the nanoparticles. The solid matrix of NLC decelerated the release following the Stokes-Einstein law of diffusion ${ }^{22)}$. The Higuchi model is normally used to describe the release profile of drugs ${ }^{26)}$. In this study, the Higuchi model could be applied to $1 \% \mathrm{w} / \mathrm{w}$ GO-loaded NLC formulation. The faster release was obtained at the first eight hours, especially for the first two hours. Then, the prolonged release was observed. However, for extract-loaded NLC formulation and $0.01 \% \mathrm{w} / \mathrm{w}$ GOloaded NLC formulation, the release profiles did not display to follow the Higuchi model. This could be explained by the small amount of $\gamma$-oryzanol in both formulations or the releasing time was not long enough to figure out if these release profiles followed the model. The release profiles of both formulations showed lower for the first two hours and increased almost constantly for $24 \mathrm{~h}$. This suggested that no enrichment of $\gamma$-oryzanol in the outer shell of the NLC particles and the release profiles only presented the prolonged release of $\gamma$-oryzanol from the inside of the particles.

\section{CONCLUSION}

Extraction of Leum Pua glutinous rice bran by using hexane as extraction solvent gave the highest percent of $\gamma$-oryzanol, although methanol gave the highest percent yield of rice bran oil. This was due to the polarity of hexane that was suitable for dissolving $\gamma$-oryzanol more than isopropanol and methanol. Moreover, methanol extract had less variety of lipid compositions compared to isopropanol extract and hexane extract. After preparing NLC formulations, it found that the particle size of extract-loaded NLC was slightly higher than GO-loaded NLC. Nevertheless, both NLC formulations were stable at $4^{\circ} \mathrm{C}$ and room temperature for at least 3 months. The encapsulation efficiency of all formulations was almost 100\% due to high lipophilicity of $\gamma$-oryzanol. The release profile of extract-loaded NLC formulation showed slightly higher than GO-loaded NLC formulation. This might be explained by various lipid components in the extract compared to GO-loaded NLC that had only two types of lipids. However, both release profiles did not follow the Higuchi model because of the small amount of $\gamma$-oryzanol in NLC formulations. Also, the burst release was not found for both formulations.

\section{ACKNOWLEDGMENTS}

This research was financially supported by the research grants (CRP5805020590) from National Research Council of Thailand (NRCT) and Agricultural Research Development Agency (Public Organization). The authors are grateful to Faculty of Engineering and Faculty of Pharmacy, Mahidol University for facility support.

\section{CONFLICT OF INTEREST}

The authors declare no conflicts of interest.

\section{REFERENCES}

1) Müller, R.H.; Shegokar, R.; Keck C.M. 20 years of lipid nanoparticles (SLN \& NLC): present state of development \& industrial applications. Curr. Drug Discovery Technol. 8, 207-227(2011).

2) Fang, C-L.; Al-Suwayeh, S.A.; Fang, J-Y. Nanostructured lipid carriers (NLCs) for drug delivery and targeting. Recent Pat. Nanotechnol. 7, 41-55 (2013) .

3) Müller, R.H.; Radtke, M.; Wissing, S.A. Nanostructured lipid matrices for improved microencapsulation of drugs. Int. J. Pharm. 242, 121-128(2002).

4) Rogers, E.; Rice S.; Nicolosi, R.; Carpenter, D.; McClelland, C.; Romanczyk, L. Identification and quantitation of $\gamma$-oryzanol components and simultaneous assessment of tocols in rice bran oil. J. Am. Oil Chem. Soc. 70, 301-307 (1993).

5) Xu, Z.; Godber, J.S. Purification and identification of components of $\gamma$-oryzanol in rice bran oil. J. Agric. Food Chem. 47, 2724-2728(1999).

6) Hiramitsu, T.; Armstrong, D. Preventive effect of antioxidants on lipid peroxidation in the retina. Ophthalmic Res. 23, 196-203(1991).

7) Cicero, A.; Gaddi, A. Rice bran oil and $\gamma$-oryzanol in the treatment of hyperlipoproteinaemias and other conditions. Phytother. Res. 15, 277-289 (2001).

8) Nanua, J.; McGregor, J.; Godbert, J. Influence of highoryzanol rice bran oil on the oxidative stability of whole milk powder 1. J. Dairy Sci. 83, 2426-2431 (2000).

9) Coppini, D.; Paganizzi, P.; Santi, P.; Ghirardini, A. Capacità protettiva nei confronti delle radiazioni solari di derivati di origine vegetale. Cosmet. News 136, 15-20 (2001).

10） Boonsit, P.; Pongpiachan, P.; Julsrigival, S.; Karladee, D. Gamma oryzanol content in glutinous purple rice landrace varieties. CMUJ Nat. Sci. 9, 151-157(2010).

11) Oliveira, R.; Oliveira, V.; Aracava, K.K.; Rodrigues, C.E.D.C. Effects of the extraction conditions on the yield and composition of rice bran oil extracted with 
ethanol-A response surface approach. Food Bioprod. Process. 90, 22-31(2012).

12) Yoshie, A.; Kanda, A.; Nakamura, T.; Igusa, H.; Hara, S. Comparison of $\gamma$-oryzanol contents in crude rice bran oils from different sources by various determination methods. J. Oleo Sci. 58, 511-518(2009).

13) Lai, P.; Li, K.Y.; Lu, S.; Chen, H.H. Phytochemicals and antioxidant properties of solvent extracts from Japonica rice bran. Food Chem. 117, 538-544(2009).

14) Hu, W.; Wells, J.H.; Shin, T-S.; Godber, J.S.; Comparison of isopropanol and hexane for extraction of vitamin $\mathrm{E}$ and oryzanols from stabilized rice bran. J. Am. Oil Chem. Soc. 73, 1653-1656 (1996).

15) Zigoneanu. I.; Williams. L.; Xu. Z.; Sabliov. C. Determination of antioxidant components in rice bran oil extracted by microwave-assisted method. Bioresour. Technol. 99, 4910-4918(2008).

16) Abidi, S.; Thiam, S.; Warner, I. Elution behavior of unsaponifiable lipids with various capillary electrochromatographic stationary phases. J. Chromatogr. A. 949, 195-207 (2002).

17) Xu, Z.; Godber, J.S. Antioxidant activities of major components of $\gamma$-oryzanol from rice bran using a linoleic acid model. J. Am. Oil Chem. Soc. 78, 645-649 (2001).

18) Shah, R.; Eldridge, D.; Palombo, E.; Harding, I. Optimisation and stability assessment of solid lipid nanoparticles using particle size and zeta potential. J. Phys. Sci. 25, 59-75 (2014).

19) Kaur, I.P.; Bhandari, R.; Bhandari, S.; Kakkar, V. Potential of solid lipid nanoparticles in brain targeting. $J$.
Controlled Release 127, 97-109(2008).

20) Das, S.; Ng, W.K.; Tan, R.B.H. Are nanostructured lipid carriers (NLCs) better than solid lipid nanoparticles (SLNs): Development, characterizations and comparative evaluations of clotrimazole-loaded SLNs and NLCs? Eur. J. Pharm. Sci. 47, 139-151(2012).

21) Martins, S.; Tho, I.; Souto, E.; Ferreira, D.; Brandl, M. Multivariate design for the evaluation of lipid and surfactant composition effect for optimisation of lipid nanoparticles. Eur. J. Pharm. Sci. 45, 613-623 (2012).

22) Teeranachaideekul, V.; Souto, E.B.; Junyaprasert, V.B.; Müller, R.H. Cetyl palmitate-based NLC for topical delivery of Coenzyme Q 10-Development, physicochemical characterization and in vitro release studies. Eur. J. Pharm. Biopharm. 67, 141-148(2007).

23) Sato, K. Crystallization behaviour of fats and lipids-a review. Chem. Eng. Sci. 56, 2255-2265 (2001).

24) Bunjes, H.; Westesen, K.; Koch, M.H. Crystallization tendency and polymorphic transitions in triglyceride nanoparticles. Int. J. Pharm. 129, 159-173 (1996).

25) Ruktanonchai, U.; Limpakdee, S.; Meejoo, S.; Sakulkhu, U.; Bunyapraphatsara, N.; Junyaprasert, V.; Puttipipatkhachorn, S. The effect of cetyl palmitate crystallinity on physical properties of gamma-oryzanol encapsulated in solid lipid nanoparticles. Nanotechnology 19, 1-10 (2008).

26) Dash, S.; Murthy, P.N.; Nath, L.; Chowdhury, P. Kinetic modeling on drug release from controlled drug delivery systems. Acta Pol. Pharm. 67, 217-223 (2010). 